\title{
Poly( $\varepsilon$-Caprolactone) and Poly (L-Lactic-Co-Glycolic Acid) Degradable Polymer Sponges Attenuate Astrocyte Response and Lesion Growth in Acute Traumatic Brain Injury
}

\author{
DARICE Y. WONG, ${ }^{1}$ SCOTT J. HOLLISTER, ${ }^{1-3}$ \\ PAUL H. KREBSBACH, ${ }^{4}$ and CHRISTOPHER NOSRAT ${ }^{4, *}$
}

\begin{abstract}
This study evaluated the response of rat brain to 2 degradable polymers (poly (L-lactic-co-glycolic acid) (PLGA), and poly( $\varepsilon$-caprolactone) (PCL)), two common materials in tissue engineering. PLGA has been extensively studied in the brain for controlled drug release as injectable microspheres and is generally accepted as biocompatible in that capacity. Biocompatibility in other forms and for different functions in the brain has not been widely studied. PCL was chosen as an alternative to PLGA for its slower degradation and less-acidic $\mathrm{pH}$ upon degradation. Porous scaffolds were made from both polymers and implanted into rat cerebral cortex for 1 and 4 weeks. Morphology, defect size, activation of microglia (OX-42) and astrocytes (glial fibrillary acidic protein (GFAP)), infiltration of activated macrophages (major histocompatibility complex (MHC)-II), and ingrowth of neurons ( $\beta$-tubulin type III (Tuj-1)) and progenitor cells (nestin) were analyzed using hematoxylin and eosin staining and immunofluorescence. PCL induced a lower inflammatory response than PLGA, as demonstrated by lower MHC-II and GFAP expression and greater ingrowth. Both polymers alleviated astrocytic activation and prevented enlargement of the defect. Tuj-1-, nestin-, and GFAP-positive cells were observed growing on both polymers at the peripheries of the sponge implants, demonstrating their permissiveness to neural ingrowth. These findings suggest that both polymers attenuate secondary death and scarring and that PCL might have advantages over PLGA.
\end{abstract}

\section{INTRODUCTION}

B RAIN LESIONS can result from a range of causes, from traumatic injury to disease. The resultant voids are unable to retain or support injections of suspension treatments such as cells ${ }^{1}$ or growth factors, which disperse quickly and lose effectiveness. From a tissue-engineering standpoint, a delivery vehicle that fills the void and contains drugs, neurotrophic factors, or cells has the potential to localize and maintain these treatments in the implant site, increasing the effectiveness, and provide a path toward repair and recon- stitution of the lost parenchyma that is lacking in current clinical treatments. ${ }^{2}$ This delivery vehicle must satisfy certain design criteria, such as permissiveness to neural cell growth and immunological compatibility. Such scaffolds should not cause significant inflammatory responses that may exacerbate the injury. Also, mechanically, the material must be close enough to the tissue dynamics that surgical insertion and normal movements of the patient do not cause more physical damage.

Treatment strategies for brain injuries such as hypoxic ischemia models focus mainly on combination treatments

\footnotetext{
Departments of ${ }^{1}$ Biomedical Engineering, ${ }^{2}$ Mechanical Engineering, ${ }^{3}$ Neurosurgery, and ${ }^{4}$ Biologic and Materials Sciences, University of Michigan at Ann Arbor, Ann Arbor, Michigan.

*Current affiliation: University of Tennessee Health Science Center, College of Dentistry, Cancer Research Building, Memphis, Tennessee.
} 
using cells or neurotrophic factors. ${ }^{3-5}$ Studies focusing on combination treatments often overlook the potential importance of the material itself and often compare improvements and outcomes with a completely empty control site rather than the untreated material. Different materials, such as gels and polymer fiber meshes, have been studied separately. Unfortunately, there are no good comparisons of materials or any in-depth study of the relative biocompatibilities of the materials themselves. To provide an optimal outcome, it would be important to study and determine the best combination of biologic therapy and material vehicles in a systematic approach.

Poly (L-lactic-co-glycolic acid) (PLGA) and its related components are among the most widely investigated degradable polymers in biomedical applications. The most extensive work with PLGA regarding material-brain research has been in controlled drug-release applications in the form of microspheres injected into the brain. ${ }^{6-8}$ PLGA has been generally accepted as biocompatible in that capacity. Poly(e-caprolactone) (PCL) has also been studied in the brain for controlled-release microspheres, ${ }^{8,9}$ although not as extensively as PLGA. PCL has been most commonly studied in other tissue types and has a history of drug delivery and tissue-engineering research elsewhere in the body. ${ }^{10-12}$ Central nervous system (CNS) inflammation has also been studied in the context of electrode implants, where close contact of neuronal activity is desired. ${ }^{13}$ The current study investigated the biocompatibility of PLGA and PCL in the capacity of CNS tissue engineering. This approach requires ingrowth and close contact with regenerating neuronal structures and integration of host tissue within and around the implant. The structure of a scaffold in a defect, rather than microspheres injected into tissue, may elicit a different host response. The definition of biocompatibility here is slightly different from controlled release and implantable electrodes, although we took guidance from both areas.

Acute inflammation in various brain-injury models occurs over the course of hours to days, encompassing hemorrhage, degeneration of damaged neurons, and macrophage infiltration. By 7 days the inflammatory response has elicited secondary cell death mechanisms that result in degeneration of white matter and expansion of the original injury site. ${ }^{14,15}$ The ultimate size of the defect depends greatly on the initial insult. Thus, different CNS defects may require different standards for success. It may be useful in some cases with large defects for the scaffold to have a much longer residence time and sturdier support than most hydrogels and PLGA derivatives can offer. PCL could fill this requirement. It is possible to use PCL as a scaffold to carry cells within a hydrogel that infuses its pores, giving the implant a definite structure and longer-lasting support once the gel has dissipated and the cells are establishing a full matrix. Although the degradation rates of PLGA and PCL can be adjusted through their initial molecular weights and the ratio of lactic acid to glycolic acid in the case of PLGA, PCL may offer another benefit in the lower acidity of its degradation prod- uct, caproic acid $(\mathrm{pKa}=4.84)$, than of lactic acid $(\mathrm{pKa}=$ $3.08)$ and glycolic acid $(\mathrm{pKa}=3.83)$.

Both polymers degrade by hydrolysis of the polymer chains. The difference in their degradation rate is mostly a result of their differing hydrophobicities. PCL is more hydrophobic than PLGA and therefore is hydrolyzed more slowly. As the molecular weight decreases, and polymer chains become smaller, macrophages phagocytose these small particles. Porous implants of PCL and PLGA in a subcutaneous site were reported at 4 weeks to have lost $39 \%$ and $74 \%$ of their molecular weights, respectively. ${ }^{16}$ Additionally, PCL has been shown to have higher total cellular ingrowth than PLGA after 4 weeks and higher inflammatory cell invasion and angiogenesis subcutaneously. ${ }^{16}$ This study compared PCL with PLGA in a salt-leached sponge architecture as they relate to brain inflammation and ingrowth in an injury site. We found that PCL elicited a lower immune response than PLGA and that both polymers lowered the scarring and secondary cell death after injury than in a defect without implant.

\section{METHODS}

PCL (CAPA 6501, molecular weight (MW) 50 kDa, Solvay Chemicals, Houston, TX) and PLGA (50:50, MW $\sim 54 \mathrm{kDa}$, Birmingham Polymers, Birmingham, AL) were used in this study. Both polymers were made into sponges using solvent casting and porogen leaching with 180- to 250$\mu \mathrm{m}$ sodium chloride crystals in a Teflon mold to form 3-mmdiameter cylinders of $3 \mathrm{~mm}$ height. PCL was dissolved in acetone and PLGA in chloroform at $13 \%(\mathrm{w}: \mathrm{v})$ and dripped into salt-packed molds from both ends until no more polymer could be absorbed. Scaffolds were sterilized in $70 \%$ ethanol and then switched to sterile Hanks Balanced Salt Solution and placed on a shaker 1 day before implantation.

Female Sprague-Dawley rats approximately $250 \mathrm{~g}$ in weight were anesthetized with isofluorane, and heads were mounted in a stereotaxic device. The skulls were exposed, bregma located, and two positions marked: $3 \mathrm{~mm}$ posterior and $3.5 \mathrm{~mm}$ left or right. Holes were drilled in the skull with a 3 -mm outer-diameter trephine at 1 or both locations. Bone chips were removed, and then the same trephine, which had a marking $3 \mathrm{~mm}$ from the bottom of its burr, was used to drill $3 \mathrm{~mm}$ into the cerebral cortex. The drill was kept perpendicular to the surface of the skull and was held steady during the removal of brain tissue through the hole in the skull. PCL and PLGA sponges were inserted into the right and left cavities, respectively. Controls received no implant in either cavity. Other researchers report the lack of contralateral damage and inflammation in a similar injury model. ${ }^{17}$ To confirm this in our own studies, 7 rats received only 1 defect in the left or right hemisphere. The distribution is as follows: at the 1-week time point, 2 rats for control (no implant) both in the right hemisphere, 1 rat with PLGA in the left hemisphere; at the 4-week time point, 3 rats with PCL with 1 left 
and 2 right hemisphere implants, and 1 rat with PLGA in the left hemisphere. Tissue was examined histologically and with all the immunofluorescent inflammation markers used in the study. Once it was confirmed that the contralateral sides were not affected and there was no difference between the right and left hemispheres, these data were combined with data for rats containing 2 defects per brain for a total of 4 samples per group per time point.

At 1 week and 4 weeks, rats were perfused transcardially with $250 \mathrm{~mL}$ of $4 \%$ paraformaldehyde in phosphate buffered saline. Brains were harvested and cryoprotected in 30\% sucrose, embedded in Tissue-Tek Optimal Cutting Temperature (OCT) compound (Electron Microscopy Sciences, Hatfield, PA), then serially sectioned coronally at $14-\mu \mathrm{m}$ thickness in groups of 5 for further analysis. There was no noticeable loss of motor function due to the implantation and no noticeable difference between groups in functional behavior after the implantation. All surgery, postsurgical recovery, and euthanasia were performed according to a protocol approved by the University of Michigan Committee on the Use and Care of Animals.

Immunofluorescence was carried out using major histocompatibility complex (MHC)-II (MCA46GA, Serotec, Raleigh, NC), ${ }^{18,19}$ nestin (Rat-401, Hybridoma Bank, University of Iowa, Iowa City, IA), ${ }^{20,21} \beta$-tubulin type $\beta$ (Tuj1, MMS-435P, Covance, Berkeley, CA), ${ }^{22,23}$ glial fibrillary acidic protein (GFAP, G9269, Sigma, St. Louis, MO), ${ }^{24,25}$ and CD11b(OX-42) (MCA275G, Serotec) ${ }^{26,27}$ Primary antibodies were used with $\mathrm{Cy}$-2-conjugated secondary antibody or Streptavidin AlexaFluor-488 (Invitrogen/Molecular Probes, Carlsbad, CA) with VectaStain ABC kit and biotinylated secondary antibodies (Vector Laboratories, Burlingame, CA). Harris hematoxylin and alcoholic eosin stains were used for assessing a general overview of inflammation and morphology. Images were taken using a Spot camera and Spot Advanced software (Diagnostic Instruments, Sterling Heights, MI). All images for a montage were taken in the same sitting with the same exposure and processing settings. When possible, all images of sections with the same type of labeling were taken on the same day or 2 to 3 consecutive days in batches of 15 to 20 slides. Positive and negative control labeling was done with all primary and secondary antibodies using stock sections from undamaged rat brain to verify their specificity and check background staining. A mixture of 5\% donkey serum (Jackson ImmunoResearch Laboratories Inc., Westgrove, PA) and 3\% BSA was used for blocking nonspecific binding.

Pixel areas for MHC-II and intensities for GFAP and OX42 antibodies were calculated using programs written in Matlab (The Mathworks, Natick, MA). The programs were written to allow the user to define specific regions of interest and calculate mean grayscale values (between 0 and 255) to determine background intensity and experimental group intensities. All intensity data presented have background subtracted. Also, for MHC-II quantification, images were thresholded to exclude background areas. Then paths were drawn in Adobe Photoshop (Microsoft Corp., Redmond, WA) to encompass total sponge area and used as a mask for the scaffold area to be thresholded. Debris and halos were removed with Photoshop before calculating areas based on intensity thresholds. GFAP and OX-42 intensities were calculated from 2 neighboring $200-\mu \mathrm{m}$-wide rectangles along each side of each scaffold (Fig. 1). Region L1 was the region immediately adjacent to the defect, and L2 was the region just adjacent to L1. Each sample section had 2 L1 regions and $2 \mathrm{~L} 2$ regions, which were combined for an average. Lengths varied, and regions were chosen to exclude edge-effect fluorescence, the hippocampus, dirt particles, air bubbles, and folds. Three regions on each section were

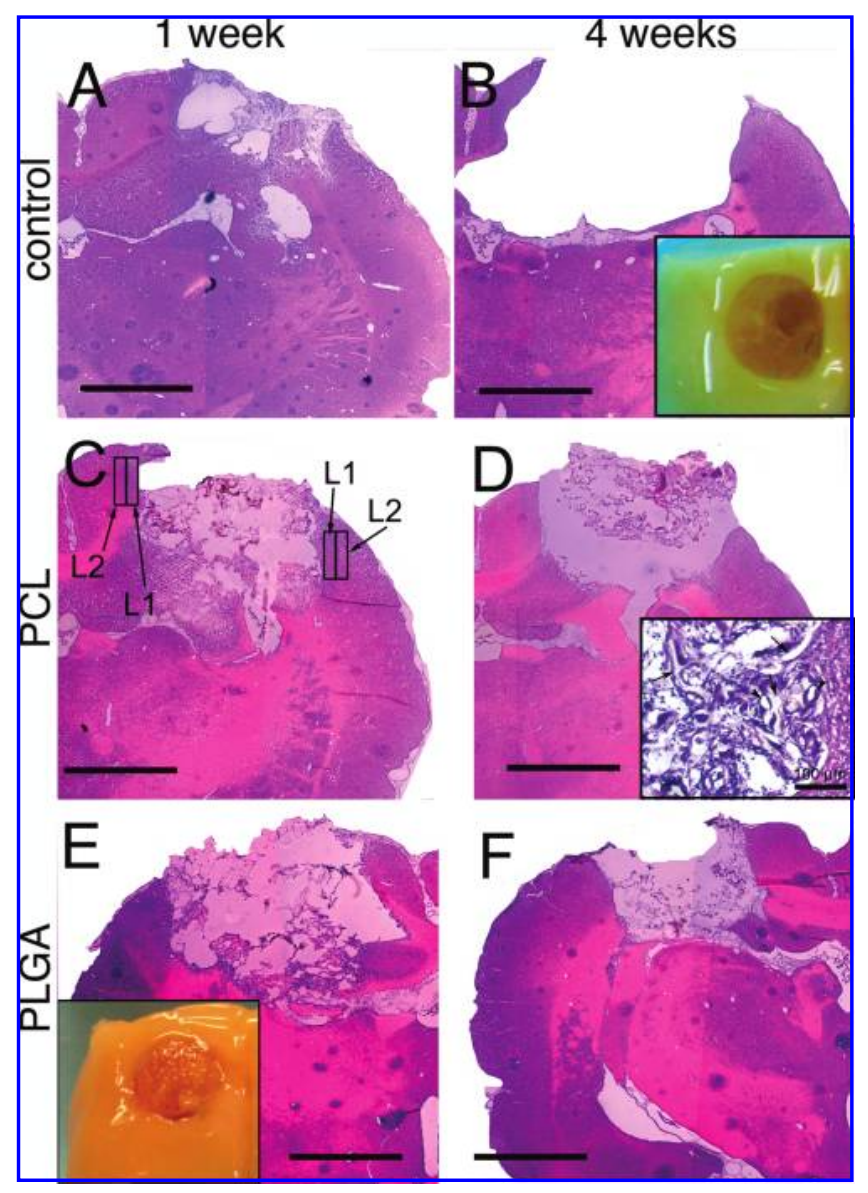

FIG. 1. Hematoxylin and eosin (H\&E)-stained sections of polymer constructs in brain cavities. (C) Regions L1 and L2 are illustrated on this section. All images (except for insets) were taken at $2 \times$ magnification and tiled together. Scale bars are $2 \mathrm{~mm}$ unless otherwise labeled. Control at (A) 1 week and (B) 4 weeks, poly(e-caprolactone) (PCL) at (C) 1 week and (D) 4 weeks, and poly (L-lactic-co-glycolic acid) (PLGA) at (E) 1 week and (F) 4 weeks (inset $\mathbf{B}$ and inset $\mathbf{E}$ ). Gross images before sectioning illustrate the visual difference between $(\mathbf{B})$ control and (E) a defect containing material (inset D). $10 \times$ mag of H\&E section showing parenchyma at the bottom corner of a defect site on the right-hand side with PCL polymer (arrows) and blood vessels (arrow heads). Color images available online at www.liebertpub.com/ten. 
chosen from areas of the cortex unaffected by the surgeries to calculate background intensities on each slide. The average total area used to calculate background was $0.09 \mathrm{~mm}^{2}$. Statistical significance was calculated using a 2-way analysis of variance (ANOVA) over time and material for MHCII areas and defect widths and 3-way ANOVA for GFAP and OX-42 intensities over time, material, and region. $\mathrm{P}$ levels below 0.05 were considered statistically significant. Tukey's least significant difference was used in post hoc tests. Error bars in graphs show standard deviations. Cellular areas within sponges were calculated from hematoxylin and eosin (H\&E)-stained sections because the polymers did not stain, whereas combined cellular and material areas were calculated from immunofluorescence images due to the polymers' persistence on slides after aqueous processing and their lowlevel autofluorescence. Defect sizes were measured using the measuring tool in Adobe Photoshop. Serial sections were measured until the middle of the defect was found, and that measurement was used. Distances were measured across the top and across the middle as parallel lines, perpendicular to the apparent angle of entry for the axis of the cylinder sponges.

\section{RESULTS}

Defects in control brains without any polymer implant increased in size over the course of the experiment, whereas PCL and PLGA helped to maintain the defect close to its original size. Brain tissue encompassed the sponges rather than shrinking away from it (Fig. 1E inset). By measuring serial sections to find the middle of each scaffold, the defect widths revealed no significant difference between the 2 polymers but were significantly different from the controls. Both polymers were able to maintain the defect sizes at approximately the original size, whereas the control defect expanded to more than $4 \mathrm{~mm}$ in diameter (Fig. 1B inset and Fig. 2A). From 2-way ANOVA over group and time, only the interaction had significance $(p p=.02)$. From the post $h o c$ analysis, there was no difference between the 2 polymers at either time point. Only the control group was significantly larger than the materials at 4 weeks. At 4 weeks, a noticeable difference in color and morphology appeared between PCL and PLGA sponges (Fig. 1). After perfusion, PCL sponges were the same color as the surrounding brain tissue, and PLGA sponges had a brownish red color that was consistent in all replicates. This may be a reflection of vascular connectivity within the sponges allowing blood to be perfused more thoroughly in PCL than PLGA sponges.

Coronal sections through the brains were used to observe the polymer sponge structure in the brain defect (Fig. 1). Shapes reminiscent of salt crystals were evident. Groups at 1 week looked less empty, as fibrin clots remained. By 4 weeks, clots cleared, but some peripheral tissue integration remained. Cells were seen in close contact with polymers, and some vascularization was seen (Fig. 1D inset). Ad-
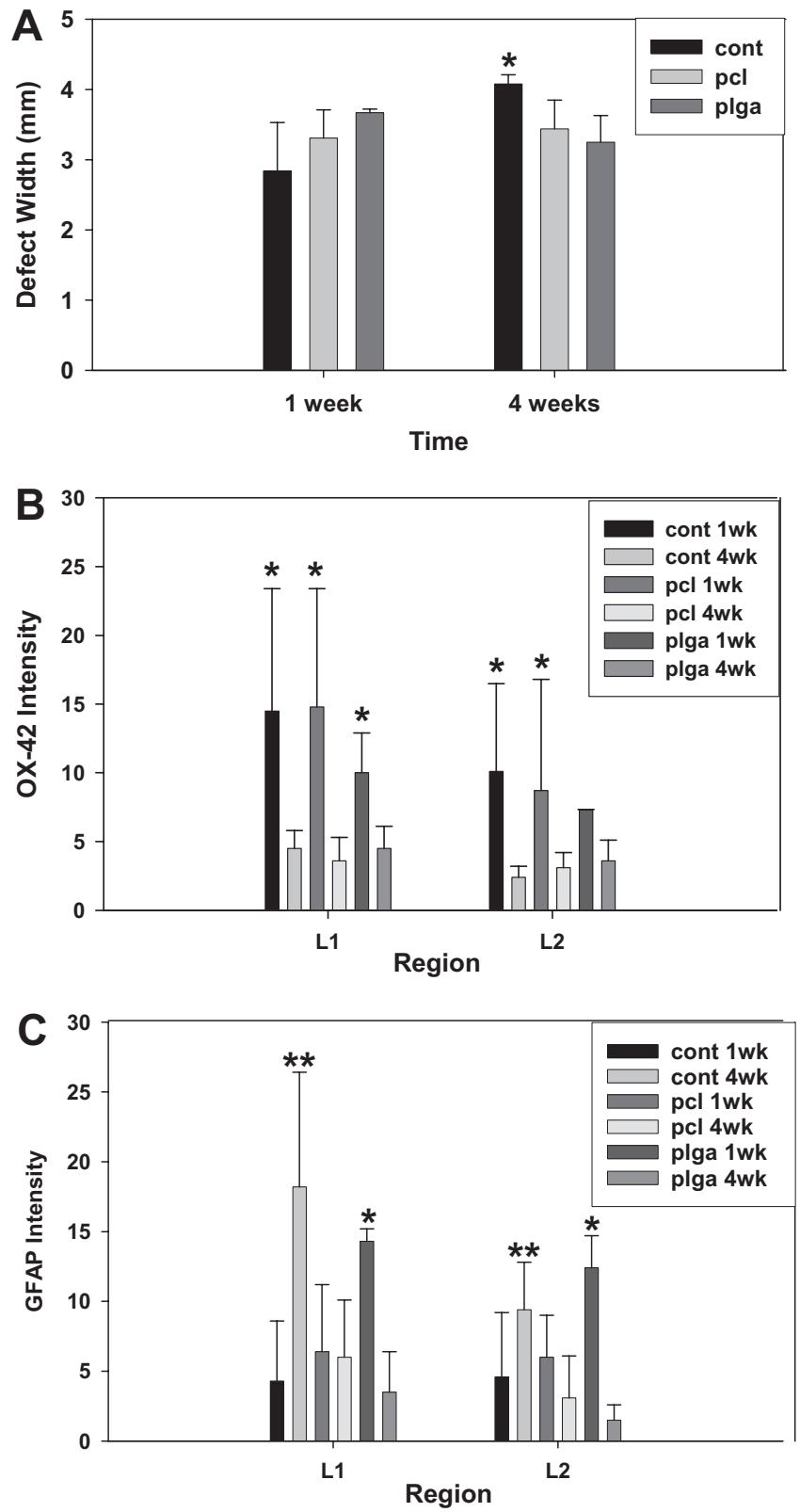

FIG. 2. Quantitative data comparing all three groups from image analysis. (A) Defect widths for each group at 1 and 4 weeks. Starred control is significantly larger than all other groups and times $(p<$ .05). (B) OX-42 intensities after background subtraction. Starred groups were not different from each other but were significantly higher than the unstarred groups. Unstarred groups were not significantly different from each other. (C) Glial fibrillary acidic protein intensities after background subtraction. All starred groups are higher than unstarred groups. The 2 doubly starred groups are significantly different from each other. Unstarred groups are not significantly different from each other (see text for detailed $p$-values).

ditionally, at 4 weeks, sponges began to degrade and had overall smaller volumes. In the H\&E images, polymers were dissolved by the xylenes during staining, leaving white areas on the slides. Occasionally, sponges were dislodged from the brains during removal of the skull, as fibrous tissue filled in 
the bony defect in the skull and formed a slight attachment between the skull and the tops of the sponges (Fig. 1D). Specimens were carefully detached from the skull and replaced in the defect cavity, but on soaking in OCT and freezing for sectioning, it was evident from their floating position relative to the rest of the brain tissue that they had moved. When evaluating the defect size, the walls of the cavity were measured. When evaluating the areas within the scaffold, a visually verified approximation of the defect surrounding the scaffold was used.

MHC-II-positive cells were present within both polymers to different degrees. The difference was statistically significant for material $(p p=.02)$ but not over time $(p p=.12)$. MHC-II-positive areas were greater in PLGA than PCL $(1.2 \%$ vs $0.5 \%)$ at the 1 -week acute phase of inflammation and did not change over time in PLGA. An increase in the mean MHC-II expression levels found in PCL (from $0.5 \%$ to $0.8 \%$ ) over time was not statistically significant and did not surpass the mean level in PLGA at either time point.

The cellular area in both materials measured from H\&E images increased significantly over time $(p p=.000)$, roughly doubling from approximately $12 \%$ to approximately $25 \%$, with a trend of PCL having 5\% to $10 \%$ higher cellular ingrowth than PLGA ( $p=.07)$, whereas the combined cellular and material area decreased over time by approximately one third $(p=.02)$, from approximately $45 \%$ to approximately $30 \%$, with no significant difference in material $(p=.97)$. This difference reflects the degradation of materials at a higher rate than ingrowth and accounts for the overall decrease in non-void space within the implant.

In analyzing inflammation peripheral to the defect cavity, OX-42 and GFAP intensities were measured in the L1 and L2 regions (Fig. 1C). For OX-42 (activated microglia), $p$-values from 3-way ANOVA and interactions were as follows: group $=.7253$, time $=0$, region $=0.0603$, group + time $=0.5159$, group + region $=0.9649$, time + region $=$ 0.2228 (Fig. 2B). Astrocytic activation was measured according to GFAP intensities in regions $\mathrm{L} 1$ and $\mathrm{L} 2$, and $p$-values from 3-way ANOVA and interactions were as follows: group $=.0001$, time $=0.0889$, region $=0.0469$, group + time $=0.0003$, group + region $=0.6454$, time + region $=0.2149$ (Fig. 2C).

OX-42-positive activated microglia were observed in control defects at 1 week (Fig. 2B) and declined by 4 weeks, reflecting a decrease in microglial activation. There was also a concomitant increase over time of GFAP-positive cells, consistent with other brain injury models. ${ }^{17,28,29}$ In contrast to the control, polymer sponge groups showed a decreasing trend over time from 1 to 4 weeks for GFAP-positive activated astrocytes, and 3-way ANOVA showed that the 3 material groups (including control) had significant differences, indicating that there was a decrease in GFAP from region L1 to L2. The 2 polymer groups had similar microglial activation $(\mathrm{OX}-42)$ to that of the controls in region $\mathrm{L} 1$, decreasing over time. However, the PLGA group had slightly lower activation of microglia, which became significant

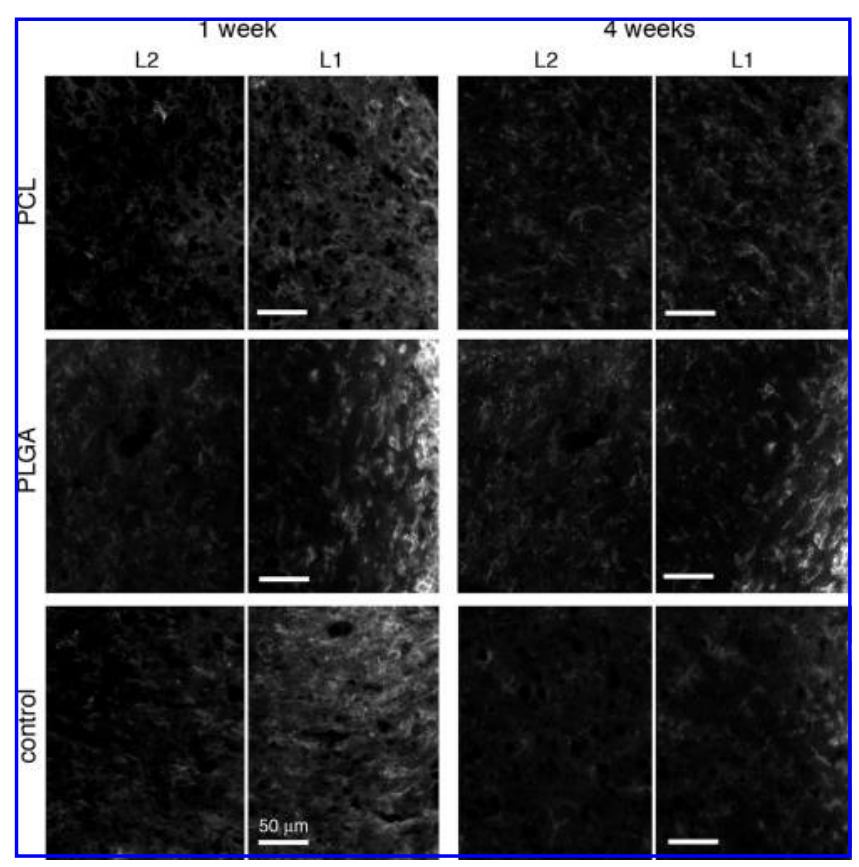

FIG. 3. OX-42 immunohistochemistry. Images of immediately adjacent regions L2 and L1 with defects located on the right. $10 \times$ magnification, scale bars, $50 \mu \mathrm{m}$.

only in region L2. Representative images of microglial morphology adjacent to the defect site in region $\mathrm{L} 1$ and further away in region L2 are shown in Figure 3. Highly activated microglial cells appear to have large bright cell bodies and short fibers, whereas unactivated microglia have more-diffuse fibrous labeling and no large cell bodies. GFAP expression around both polymers was less than around the control after 4 weeks and did not differ from that of each other at that late time point. However, in contrast to microglial activation, PLGA groups had significantly higher levels of GFAP than PCL at 1 week, and the PCL groups were not significantly changed over time (Fig. 2C, and Fig. 4). Cytokines from the activated microglia activate astrocytes, ${ }^{30}$ leading to increased production of GFAP. GFAP levels reflect their activation level, the highest of which can indicate glial scar formation. GFAP-labeled astrocytes with long thin fibers activated next to the polymer-implanted defects contrast the control regions at 4 weeks, which had higher activation based on GFAP intensities (Fig. 2C) and show astrocytes close to the defect border aligning and densely entangling their fibers (Fig. 4).

Ingrowth of GFAP-positive astrocytes into the sponges was seen in both polymers to occur from the bottom and edges up toward the middle, whereas MHC-II-positive cells were seen distributed throughout the sponges. This suggests astrocyte migration into the scaffolds from host tissue and MHC-II-infiltrating cells from peripheral blood residing in all areas of the sponge from the initial absorption. In the bottom corners of the sponges, close to their contact with the lateral ventricles, nestin-positive and Tuj-1-positive 


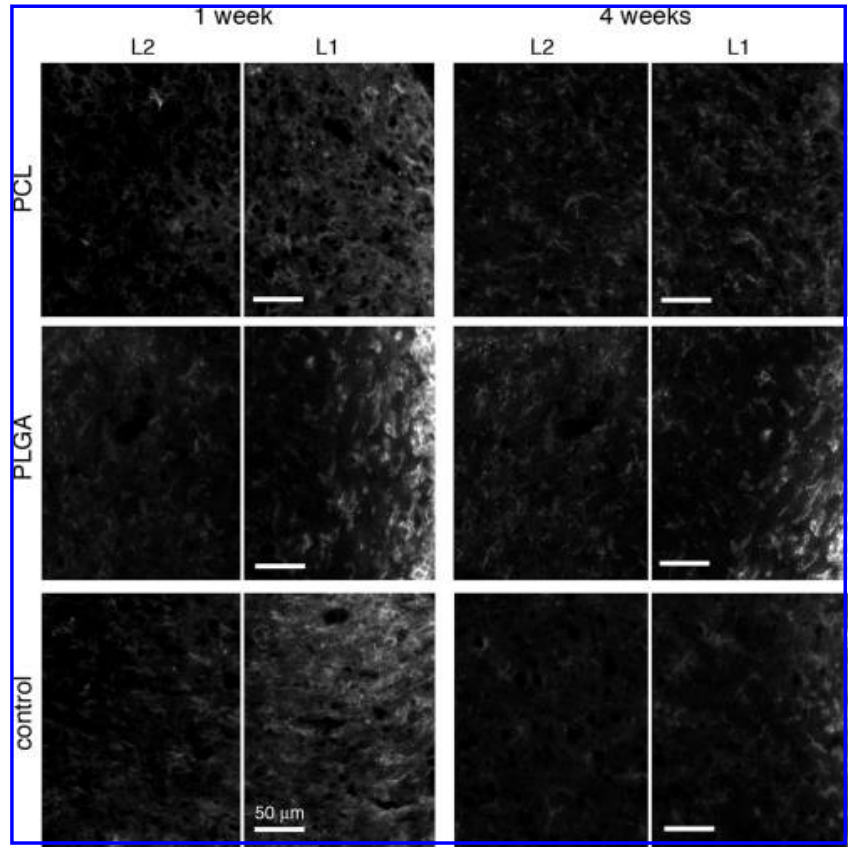

FIG. 4. Glial fibrillary acidic protein immunohistochemistry. Images of immediately adjacent regions L2 and L1 with defects located on the right. $10 \times$ magnification, scale bars, $50 \mu \mathrm{m}$.

cells were found in close contact with the materials after 4 weeks (Fig. 5).

\section{DISCUSSION}

In brief summary, porous PCL and PLGA implants were able to decrease cell death and support neural cell growth in vivo, an improvement over no implant use. Neither polymer caused any severe inflammation, actually modifying the response such that the environment was more amenable to regeneration. Both polymers are suitable for use as a delivery vehicle of treatments, but PCL may provide advantages over PLGA depending on the treatment strategy.

The defect width of the control group over time was smaller at 1 week than that of the polymer groups and grew to be larger at 4 weeks. Because all of the defects (control and implant) were first created in the same way, the surgical preparation was not a factor. The only difference would be that the polymer was placed inside the cavity. It is possible that, because of swelling, the control defects might become smaller because there was no polymer to keep the walls of the defect from caving in, which we consider more likely than caving out while still contained in vivo. With regard to analytical preparation, all brains were fixed by perfusion before any physical manipulation of the tissue. Upon dissection from the skull, it was apparent on visual inspection that the sizes of defects had grown in the control. Brains were frozen for sectioning in large pieces, with plenty of tissue surrounding the defect site to hold the walls in their original shape. In addition, most often it was the deeper

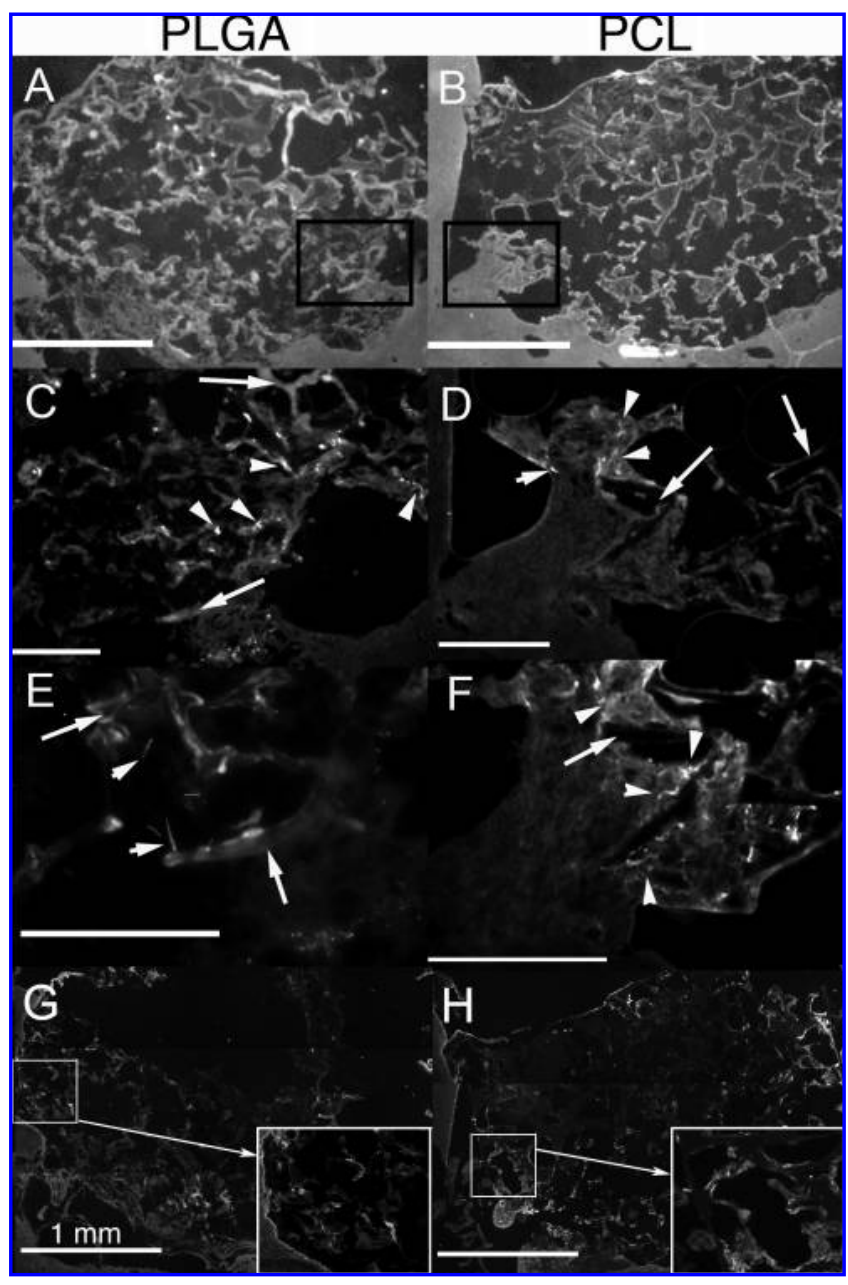

FIG. 5. Representative immunofluorescent images identifying neural cells. Top 2 images are provided for perspective in (A) poly (L-lactic-co-glycolic acid) (PLGA) and (B) poly(e-caprolactone) (PCL) sponges at 4 weeks, no specific labeling. Boxes indicate corresponding regions for $\mathbf{C}, \mathbf{D}, \mathbf{E}$, and $\mathbf{F}$. Nestin-labeled cells (arrowheads) in (C) PLGA and (D) PCL in close proximity to polymer materials (long arrows). $\beta$-tubulin type III-labeled cells (arrowheads) in (E) PLGA and (F) PCL interact with polymers (long arrows). Glial fibrillary acidic protein-labeled astrocytes in (G) PLGA and (H) PCL distributed within the sponges have highly fibrous labelling (insets to $\mathbf{G}$ and $\mathbf{H}$ ). Scale bars in A, B, G, $\mathrm{H}, 1 \mathrm{~mm}$. Scale bars in C, D, E, F, $300 \mu \mathrm{m}$.

regions of the defect, not the top edges, that showed the greatest increase in width (Fig. 1B). It is clear from the histology in the remaining tissue that cell death, and not simple physical expansion during preparation of the section for histology, was the major cause of differences in defect width. Delayed enlargement of defects due to secondary cell death is also a known, common result of traumatic brain injury under several injury models. ${ }^{14,15}$ The mechanism of the attenuation of this cell death by the polymers in this study is unknown. Polyethylene glycol (PEG) has been shown to offer neuroprotective effects, although the mechanism is also unknown. ${ }^{31,32}$ Speculation on PEG interactions with 
proteins and mitochondria because of its ability to enter the cytosol could possibly provide some of the reasons that PCL and PLGA offered some benefit over no polymer implant or why there was a small benefit from PCL over PLGA, because these polymers differ slightly in $\mathrm{pH}$ and structure. Their phagocytosis by macrophages is a pathway into the cell that may be similar to the pathway that nanoparticles of PEG take. ${ }^{31,32}$ These possibilities remain to be investigated.

It was observed during the surgeries that implantation of the sponges decreased bleeding, similar to the effect from applying gauze or cotton during the surgeries to stop the initial bleeding, but the implants were never removed, as cotton was from the control groups, before closing. Macrophages $\left(\mathrm{CD}^{+}\right)$and microglia have been shown to be a mostly blood-derived subset of MHC-II- or ED1-reactive macrophages and microglia that reside mostly in the border of pan-necrosis in contusion injury. These cells may play a large role in lesion progression. ${ }^{33}$ It is likely that the MHCII-positive cells in the scaffolds of this study, which are mostly blood derived, contain a $\mathrm{CD} 8^{+}$cell population, but the presence of a biomaterial scaffold may have played a role in decreasing tissue damage by leading those cells away from the border of the defect or preventing them from reaching the parenchyma from the circulation by acting as a barrier and trap for infiltrating cells. It is possible that this passive prevention by the biomaterials was able to save some of the parenchyma from delayed death and to reduce the need for the parenchyma to form a glia limitans.

Both polymers allowed ingrowth of cells, although PCL showed a slightly higher rate than PLGA. Many of these cells inside the scaffolds were macrophages. As PCL and PLGA hydrolytically degrade, macrophages phagocytosed smaller-molecular-weight segments of the polymers. ${ }^{12}$ The slower rate of hydrolytic degradation in PCL may have led to the lower level of macrophages (MHC-II labeling) than with PLGA in the material scaffold for clean-up of degradation products. The higher total cellular ingrowth in PCL than in PLGA has also been seen subcutaneously for these materials, supposedly because of the difference in initial $\mathrm{pH}$ of the polymers and from their degradation rates, but the mechanism has not been fully elucidated. ${ }^{16}$

Some of the infiltrating cells of the scaffolds other than macrophages were likely fibroblasts and astrocytes and possibly microglia from the cerebral cortex, although the microglia cannot be separately identified from circulating macrophages and neutrophils. However, astrocytes and microglia found in the parenchyma can be used to gauge levels of internal inflammation in the brain. Data at the early time point reflected lower microglial activation (OX-42) in PLGA groups than in PCL and control but a lower astrocytic activation (GFAP) in PCL than in PLGA and controls. The contrasts in astrocytic and microglial activation between polymers may be due to a time course difference in the first few weeks, converging by the fourth week. However, microglia have been shown to play neuroprotective roles, ${ }^{34}$ which might explain why a higher microglial activation in the PCL group led to lower astrocytic activation than in the PLGA group. Microglial activation may mediate astrocytic activation and the astrocyte's propensity to form a glial scar. Regardless, the difference was short lived, and both polymers had similar astrocytic activation by 4 weeks, which was lower than in the control group. Their earlier differences may manifest in a difference in neuronal damage in other areas of the brain, but this effect was not investigated, and the overall differences in pathology were small. These lower levels of GFAP suggest that polymer sponges not only impede the growth of the defect size, but could also attenuate the amount of scarring that could occur in surrounding brain tissue, whereas the increase over time in the control group suggests the standard formation of a glia limitans. The lower astrocytic activity in polymer groups than in the control group may be linked to the ability of cells to migrate into the polymers, because there is less of a cellular barrier.

Because the degradation rates of the polymers are based primarily on a constant factor, hydrophobicity, one can assume that at longer time points one would still compare inflammations in response to both polymers' molecules being released from their bulk forms at their individual release rates. The time point in this study of 4 weeks was not long enough to track the entire response over a majority of the degradation for both polymers. However, the time points chosen here reflect important points for comparison. The general time course of acute inflammation is within the first week, after which chronic inflammation sets in and remains relatively constant. Macrophages infiltrate a brain injury within the first week. ${ }^{14,15}$ Interventions to prevent secondary cell death are needed during the acute phase. Thus if there are differences in the tissue response elicited by the polymers, the most pivotal, such as secondary cell death and cellular migration, would have occurred in the first week and be evident by 4 weeks. Longer time points would likely amplify the differences or dampen them. It is, however, possible that a sudden change in the release of polymer products could occur toward the end, when the last portion of polymer might reach the small molecular weight needed to be phagocytosed all at once. If this is the case, it is likely that PCL, degrading more slowly yet being taken in by macrophages in the same way as PLGA, would cause less drastic a change. It is unlikely that longer time points would change the overall working conclusion of this study, that both PCL and PLGA can be used for implantation in the brain.

Although there was not extensive infiltration of the polymer scaffolds by nestin- and Tuj-1-positive cell types at 4 weeks, it is not unexpected, because these implants contained no bioactive treatment or cells. The neighboring subependymal zone could be a source of progenitor cells entering the defect site and contacting or entering the scaffolds, based on the location of the few cells that were observed and evidence in the literature of nestin-positive cells from the subependymal layer migrating toward lesions. ${ }^{17,35}$ Although there can be no comparison of relative permissiveness of the 2 polymers to progenitor and neuronal 
ingrowth from the data, they can both be deemed permissive. It is likely that other cell types in the scaffold surrounding the few nestin- and Tuj-1-positive cells are astrocytes and could play a supportive role in allowing those nestin- and Tuj-1-positive cells to interact with the sponges (Fig. 5). Longer time points would be helpful in future studies to determine any differences in support of neural cell ingrowth between the polymers. It is plausible that additional experimental biological factors such as stem cells or growth factors may play a much larger role than any differences between the polymer materials for this type of interaction.

\section{CONCLUSIONS}

Lesions in the brain benefit from the presence of polymeric scaffolds and will likely be more effectively treated with drug, growth factor, or stem cell delivery in the scaffolds. PCL and PLGA should be considered to be biocompatible and beneficial for traumatic brain injury in sponge scaffold form. From the data on ingrowth and infiltrating inflammatory cells, combined with its longer residence time, PCL may have an advantage over PLGA for future studies.

\section{ACKNOWLEDGMENTS}

The authors would like to thank Dr. K. Holmberg for advice on techniques and helpful discussions and Steve Gross for his help with the preliminary surgeries. Funding provided by National Institute of Dental and Craniofacial Research Grant DE007057.

\section{REFERENCES}

1. Johann, V., Schiefer, J., Sass, C., Mey, J., Brook, G., Kruttgen, A., Schlangen, C., Bernreuther, C., Schachner, M., Dihne, M., and Kosinski, C.M. Time of transplantation and cell preparation determine neural stem cell survival in a mouse model of Huntington's disease. Exp Brain Res 177, 458, 2007.

2. Thompson, T.P., Lunsford, L.D., and Kondziolka, D. Restorative neurosurgery: Opportunities for restoration of function in acquired, degenerative, and idiopathic neurological diseases. Neurosurgery 45, 741, 1999.

3. Park, K.I., Teng, Y.D., and Snyder, E.Y. The injured brain interacts reciprocally with neural stem cells supported by scaffolds to reconstitute lost tissue. Nat Biotechnol 20, 1111, 2002.

4. Tate, M.C., Shear, D.A., Hoffman, S.W., Stein, D.G., and LaPlaca, M.C. Biocompatibility of methylcellulose-based constructs designed for intracerebral gelation following experimental traumatic brain injury. Biomaterials 22, 1113, 2001.

5. Tian, W.M., Hou, S.P., Ma, J., Zhang, C.L., Xu, Q.Y., Lee, I.S., Li, H.D., Spector, M., and Cui, F.Z. Hyaluronic acidpoly-D-lysine-based three-dimensional hydrogel for traumatic brain injury. Tissue Eng 11, 513, 2005.
6. Popovic, N., and Brundin, P. Therapeutic potential of controlled drug delivery systems in neurodegenerative diseases. Int J Pharm 314, 120, 2006.

7. Kou, J.H., Emmett, C., Shen, P., Aswani, S., Iwamoto, T., Vaghefi, F., Cain, G., and Sanders, L. Bioerosion and biocompatibility of poly(d,1-lactic-co-glycolic acid) implants in brain. J Control Release 43, 123, 1997.

8. Fournier, E., Passirani, C., Montero-Menei, C.N., and Benoit, J.P. Biocompatibility of implantable synthetic polymeric drug carriers: focus on brain biocompatibility. Biomaterials 24, 3311, 2003.

9. Menei, P., Croue, A., Daniel, V., Pouplardbarthelaix, A., and Benoit, J.P. Fate and biocompatibility of 3 types of microspheres implanted into the brain. J Biomed Mater Res 28, 1079, 1994.

10. Tang, Z.G., Black, R.A., Curran, J.M., Hunt, J.A., Rhodes, N.P., and Williams, D.F. Surface properties and biocompatibility of solvent-cast poly[epsilon-caprolactone] films. Biomaterials 25, 4741, 2004.

11. Taylor, M.S., Daniels, A.U., Andriano, K.P., and Heller, J. 6 Bioabsorbable Polymers - in-Vitro Acute Toxicity of Accumulated Degradation Products. J Appl Biomater 5, 151, 1994.

12. Sun, H.F., Mei, L., Song, C.X., Cui, X.M., and Wang, P.Y. The in vivo degradation, absorption and excretion of PCLbased implant. Biomaterials 27, 1735, 2006.

13. Polikov, V.S., Tresco, P.A., and Reichert, W.M. Response of brain tissue to chronically implanted neural electrodes. J Neurosci Meth 148, 1, 2005.

14. Williams, A.J., Hartings, J.A., Lu, X.C., Rolli, M.L., and Tortella, F.C. Penetrating ballistic-like brain injury in the rat: differential time courses of hemorrhage, cell death, inflammation, and remote degeneration. J Neurotrauma 23, 1828, 2006.

15. Bramlett, H.M., and Dietrich, W.D. Pathophysiology of cerebral ischemia and brain trauma: similarities and differences. J Cereb Blood Flow Metab 24, 133, 2004.

16. Sung, H.J., Meredith, C., Johnson, C., and Galis, Z.S. The effect of scaffold degradation rate on three-dimensional cell growth and angiogenesis. Biomaterials 25, 5735, 2004.

17. Chen, S., Pickard, J.D., and Harris, N.G. Time course of cellular pathology after controlled cortical impact injury. Exp Neurol 182, 87, 2003.

18. Whiteland, J.L., Nicholls, S.M., Shimeld, C., Easty, D.L., Williams, N.A., and Hill, T.J. Immunohistochemical detection of T-cell subsets and other leukocytes in paraffinembedded rat and mouse tissues with monoclonal antibodies. J Histochem Cytochem 43, 313, 1995.

19. McMaster, W.R., and Williams, A.F. Identification of Ia glycoproteins in rat thymus and purification from rat spleen. Eur J Immunol 9, 426, 1979.

20. Hockfield, S., and McKay, R.D. Identification of major cell classes in the developing mammalian nervous system. J Neurosci 5, 3310, 1985.

21. Hartfuss, E., Galli, R., Heins, N., and Gotz, M. Characterization of CNS precursor subtypes and radial glia. Develop Biol 229, 15, 2001.

22. Mozzetti, S., Ferlini, C., Concolino, P., Filippetti, F., Raspaglio, G., Prislei, S., Gallo, D., Martinelli, E., Ranelletti, F.O., Ferrandina, G., and Scambia, G. Class III beta-tubulin overexpression is a prominent mechanism of paclitaxel resistance in ovarian cancer patients. Clin Cancer Res 11, 298, 2005. 
23. Zhang, Y.W., Denham, J., and Thies, R.S. Oligodendrocyte progenitor cells derived from human embryonic stem cells express neurotrophic factors. Stem Cells Devel 15, 943, 2006.

24. Saadoun, S., Papadopoulos, M.C., Davies, D.C., Krishna, S., and Bell, B.A. Aquaporin-4 expression is increased in oedematous human brain tumours. J Neurol Neurosurg Psychiatry 72, 262, 2002.

25. Dehay, C., Savatier, P., Cortay, V., and Kennedy, H. Cellcycle kinetics of neocortical precursors are influenced by embryonic thalamic axons. J Neurosci 21, 201, 2001.

26. Jones, N.M., and Bergeron, M. Hypoxia-induced ischemic tolerance in neonatal rat brain involves enhanced ERK1/2 signaling. J Neurochem 89, 157, 2004.

27. Stidworthy, M.F., Genoud, S., Li, W.W., Leone, D.P., Mantei, N., Suter, U., and Franklin, R.J. Notch1 and Jagged1 are expressed after CNS demyelination, but are not a major ratedetermining factor during remyelination. Brain 127, 1928, 2004.

28. Aihara, N., Hall, J.J., Pitts, L.H., Fukuda, K., and Noble, L.J. Altered immunoexpression of microglia and macrophages after mild head injury. J Neurotrauma 12, 53, 1995.

29. Holmin, S., and Mathiesen, T. Biphasic edema development after experimental brain contusion in rat. Neurosci Lett 194, 97, 1995.

30. Bianco, F., Pravettoni, E., Colombo, A., Schenk, U., Moller, T., Matteoli, M., and Verderio, C. Astrocyte-derived ATP induces vesicle shedding and IL-1 beta release from microglia. J Immunol 174, 7268, 2005.
31. Luo, J., Borgens, R., and Shi, R. Polyethylene glycol improves function and reduces oxidative stress in synaptosomal preparations following spinal cord injury. J Neurotrauma 21, 994, 2004.

32. Kerwin, B.A., Chang, B.S., Gegg, C.V., Gonnelli, M., Li, T., and Strambini, G.B. Interactions between PEG and type I soluble tumor necrosis factor receptor: modulation by $\mathrm{pH}$ and by PEGylation at the $\mathrm{N}$ terminus. Protein Sci 11, 1825, 2002.

33. Zhang, Z., Artelt, M., Burnet, M., Trautmann, K., and Schluesener, H.J. Early infiltration of CD8 + macrophages/ microglia to lesions of rat traumatic brain injury. Neuroscience 141, 637, 2006.

34. Streit, W.J. Microglia as neuroprotective, immunocompetent cells of the CNS. Glia 40, 133, 2002.

35. Holmin, S., Almqvist, P., Lendahl, U., and Mathiesen, T. Adult nestin-expressing subependymal cells differentiate to astrocytes in response to brain injury. Eur J Neurosci 9, 65, 1997.

Address reprint requests to:

Darice Wong

Biomedical Engineering Dept.

2420 Lurie BME Bldg. 1101 Beal Ave. Ann Arbor, MI 48109

E-mail: dywong@umich.edu 



\section{This article has been cited by:}

1. Sven Möllers, Ingo Heschel, Leon H.H. Olde Damink, Frank Schügner, Ronald Deumens, Barbara Müller , Ahmet Bozkurt , Jose Gerardo Nava , Johannes Noth , Gary A. Brook . 2009. Cytocompatibility of a Novel, Longitudinally Microstructured Collagen Scaffold Intended for Nerve Tissue RepairCytocompatibility of a Novel, Longitudinally Microstructured Collagen Scaffold Intended for Nerve Tissue Repair. Tissue Engineering Part A 15:3, 461-472. [Abstract] [Full Text] [PDF] [PDF Plus]

2. Jose Gerardo-Nava, Tobias Führmann, Kristina Klinkhammer, Nadine Seiler, Jörg Mey, Doris Klee, Martin Möller, Paul D Dalton, Gary A Brook. 2009. Human neural cell interactions with orientated electrospun nanofibers in vitro. Nanomedicine 4:1, 11-30. [CrossRef]

3. Hyun Hee Ahn, Kyung Sook Kim, Jung Hwa Lee, Ju Young Lee , Byung Soo Kim , Il Woo Lee, Heung Jae Chun , Jae Ho Kim , Hai Bang Lee , Moon Suk Kim . In Vivo Osteogenic Differentiation of Human Adipose-Derived Stem Cells in an Injectable In Situ-Forming Gel ScaffoldIn Vivo Osteogenic Differentiation of Human Adipose-Derived Stem Cells in an Injectable In Situ-Forming Gel Scaffold. Tissue Engineering Part A, ahead of print. [Abstract] [PDF] [PDF Plus]

4. Darice Y. Wong, Paul H. Krebsbach, Scott J. Hollister. 2008. Brain cortex regeneration affected by scaffold architectures. Journal of Neurosurgery 109:4, 715-722. [CrossRef] 